\title{
Study on coloring method of airport flight-gate allocation problem
}

\author{
Hongyan Li ${ }^{1}$, Xianfeng Ding ${ }^{1 *}$ (D) Jiang Lin ${ }^{1}$ and Jingyu Zhou ${ }^{2}$
}

\author{
"Correspondence: fxxd@163.com \\ ${ }^{1}$ College of Science, Southwest \\ Petroleum University, Chengdu, \\ China \\ Full list of author information is \\ available at the end of the article
}

\begin{abstract}
With the development of economy, more and more people travel by plane. Many airports have added satellite halls to relieve the pressure of insufficient boarding gates in airport terminals. However, the addition of satellite halls will have a certain impact on connecting flights of transit passengers and increase the difficulty of reasonable allocation of flight and gate in airports. Based on the requirements and data of question $\mathrm{F}$ of the 2018 postgraduate mathematical contest in modeling, this paper studies the flight-gate allocation of additional satellite halls at airports. Firstly, match the seven types of flights with the ten types of gates. Secondly, considering the number of gates used and the least number of flights not allocated to the gate, and adding the two factors of the overall tension of passengers and the minimum number of passengers who failed to transfer, the multi-objective $0-1$ programming model was established. Determine the weight vector $w=(0.112,0.097,0.496,0.395)$ of objective function by entropy value method based on personal preference, then the multi-objective 0-1 programming model is transformed into single-objective 0-1 programming model. Finally, a graph coloring algorithm based on parameter adjustment is used to solve the transformed model. The concept of time slice was used to determine the set of time conflicts of flight slots, and the vertex sequences were colored by applying the principle of "first come first serve". Applying the model and algorithm proposed in this paper, it can be obtained that the average value of the overall tension degree of passengers minimized in question $\mathrm{F}$ is $35.179 \%$, the number of flights successfully allocated to the gate maximized is 262 , and the number of gates used is minimized to be 60 . The corresponding flight-gate difficulty allocation weight is $\alpha=0.32$ and $\beta=0.40$, and the proportion of flights successfully assigned to the gate is $86.469 \%$. The number of passengers who failed to transfer was 642 , with a failure rate of $23.337 \%$.
\end{abstract}

Keywords: Flight-gate allocation; Multi-objective 0-1 programming; Entropy value method; Graph coloring

\section{Introduction}

With the rapid development of economy, more and more people travel by air, which has caused a great impact on airports whose passenger flow has reached the saturation state. Thus, most airports add satellite hall $\mathrm{S}$ in the existing terminal $\mathrm{T}$ to relieve the pressure of insufficient boarding gates in the original terminal. However, the addition of satellite hall will cause some difficulties in connecting flights of transit passengers. Thus, how to schedule and plan flights at airports, optimize the allocation of boarding gates to the greatest

(c) The Author(s) 2019. This article is distributed under the terms of the Creative Commons Attribution 4.0 International License (http://creativecommons.org/licenses/by/4.0/), which permits unrestricted use, distribution, and reproduction in any medium, provided you give appropriate credit to the original author(s) and the source, provide a link to the Creative Commons license, and indicate if changes were made. 
extent and reduce the transfer tension of transit passengers has become a hot issue in the civil aviation industry.

A stop at the gate is identified by the arrival flight and departure flight as a "departure". Flight-gate allocation is the allocation of arriving and departing flights to the appropriate gate, which is affected by several factors, such as the density of flights arriving at the station, the number of flights, the type of airframe, the stopping time, etc., and the transit passenger is the passenger who transfers from the arriving flight to the departure flight executed by the same or different planes. Terminal $\mathrm{T}$ has complete international airport terminal functions, including departure, arrival, entry and exit and waiting. Satellite hall $\mathrm{S}$ is an extension of terminal $\mathrm{T}$, which can wait for the flight and has no entry and exit functions. Terminal $\mathrm{T}$ and satellite hall $\mathrm{S}$ are both called terminal hall, and they are connected by MRT lines. The boarding gate in terminal $\mathrm{T}$ and satellite hall $\mathrm{S}$ is a fixed plane seat, whose functional properties are given in advance, and each flight can only be assigned to a boarding gate that matches its properties.

In 1984, Babic et al. [1] established the $0-1$ integer programming model of flight-gate allocation. This model takes the minimum walking distance of passengers as the objective function, and uses branch and bound algorithm to solve the model, but does not consider transit passengers in its model. In 1985, Mangoubi and Mathaisel [2] took into account the walking distance of transit passengers based on the work of Babic et al. The linear relaxation method of mixed integer programming and heuristic algorithm are used to solve the problem. The comparison shows that the performance of heuristic algorithm is nearly optimal. In 2001, Yan and Huo [3] set a fixed buffer time between successive flights assigned to the same flight location to absorb uncertain changes at flight time, and established a multi-objective $0-1$ integer programming model. However, since the density of flight take-off and landing has the law of changing at any time, the method of setting the buffer time of aircraft position to a fixed value is not very targeted. In 2005, Wen et al. [4] adopted the graph coloring model to solve the problem of flight gate allocation, and the objective function was to minimize the total number of gates occupied by flights. In the same year, Tian and Xiong [5] used genetic algorithm to solve the problem of flight gate allocation, mainly using proportional selection, two-point crossover and random variation. In order to accelerate the convergence rate, the objective function is modified by simulated annealing. In 2008, Ju and $\mathrm{Xu}$ [6] took the minimum distance required for passenger transfer as the objective function, and applied greedy simulated annealing algorithm to solve the problem of flight gate allocation. In the same year, Kong [7] took the total walking distance of passengers leaving Hong Kong and the free time of the balanced boarding gate as optimization goals, and established a multi-objective integer programming model. The target is transformed by linear weighting method and solved by genetic algorithm. In 2010, Jiang [8] established an allocation model with the optimization objectives of the minimum number of occupied parking Spaces, the shortest average travel distance of passengers and the minimum sum of squares of idle parking Spaces. Graph coloring algorithm and improved genetic algorithm are used to solve the model. In the same year, Wen [9] established a graph theory model of flight gate allocation problem by applying time slice algorithm, converted the problem into graph coloring problem, and solved it with genetic algorithm. Although the simulation results are good, the algorithm may converge to the local optimal solution at some times. In 2013, Li [10] established a network model for flight gate allocation problem, and proposed an algorithm for flight gate allocation from 
the three aspects of departure flights, inbound flights and inbound and outbound flights respectively. In 2014, Zeng et al. [11] established an allocation model, which took the minimum total walking distance of passenger and the minimum variance of average walking distance of passengers as the objective function, and achieved good results. In 2015, Wang [12] established a mixed set planning model for busy airports with the goal of maximizing the bridge ratio. This model includes rules such as model matching, aircraft towing, flight nature, and combination of aircraft positions, etc. Finally, the model and program were verified with an example.

Applying the requirements and data of question $\mathrm{F}$ of the 2018 postgraduate mathematical contest in modeling, this paper takes into account the model matching between flights and boarding gates, the conflict between flights in arrival time, departure time and stay time, the priority of parking at the aircraft gate, and the overall tension of transfer passengers. An optimal flight-gate allocation model was established from the global perspective, and the flight-gate allocation graph coloring algorithm based on parameter adjustment was used to solve the established model.

The data used to support the findings of this study are available at the question $\mathrm{F}$ of the 2018 postgraduate mathematical contest in modeling website (https://cpipc.chinadegrees. $\mathrm{cn} / \mathrm{cw} / \mathrm{hp} / 4)$.

\section{Flight-gate optimization allocation model}

\subsection{Problem description}

In the flight-gate allocation at an airport,

(1) terminal $\mathrm{T}$ has 28 gates and satellite hall $\mathrm{S}$ has 41 gates.

(2) There is a MRT line between T and S, which takes 8 minutes each way, and the gap time between two planes assigned at the same gate must be greater than or equal to 45 minutes.

(3) All gates of $\mathrm{T}$ and $\mathrm{S}$ are allocated according to the overall planning. The functional properties of each gate include domestic/international, arrival/departure, wide-body/narrow-body aircraft, etc.

(4) The arrival and departure of each aircraft must be allocated at the same gate, during which they cannot be moved elsewhere. The airport has another simple temporary seat for planes that cannot be allocated at a fixed gate to stop, assuming the number of temporary seats is unlimited.

(5) The process of transit passengers from the arrival of the previous flight to the departure of the next flight, which is combined into 16 different scenarios by domestic (D) and international (I), terminal (T) and satellite hall (S), and the shortest process time and MRT number of rides exist between these scenarios.

(6) The transfer time of passengers is the sum of the shortest process time, MRT time and travel time. It is required to minimize the number of boarding gates used when as many flights as possible are allocated to appropriate boarding gates and the minimum overall tension of transfer passengers is the primary factor.

The model of wide and narrow body aircraft, the minimum process time of transfer passengers, the number of MRT rides, the walking time of transfer passengers, the plane transfer plan of specific flights, information of transfer passengers and gate data can be obtained in the official website of the 2018 postgraduate mathematical contest in modeling $\mathrm{F}$. 
Table 1 Boarding gate classification

\begin{tabular}{lllllllllll}
\hline Boarding gate type & DDN & IIN & XXN & DXN & XDN & DDW & IIW & XXW & IXW & XIW \\
\hline Quantity & 35 & 4 & 2 & 2 & 2 & 2 & 17 & 3 & 1 & 1 \\
\hline
\end{tabular}

Table 2 Flight pair classification

\begin{tabular}{llllllll}
\hline Flight pair type & DDN & IIN & DIN & IDN & IIW & DIW & IDW \\
\hline Quantity & 145 & 28 & 45 & 36 & 39 & 5 & 5 \\
\hline
\end{tabular}

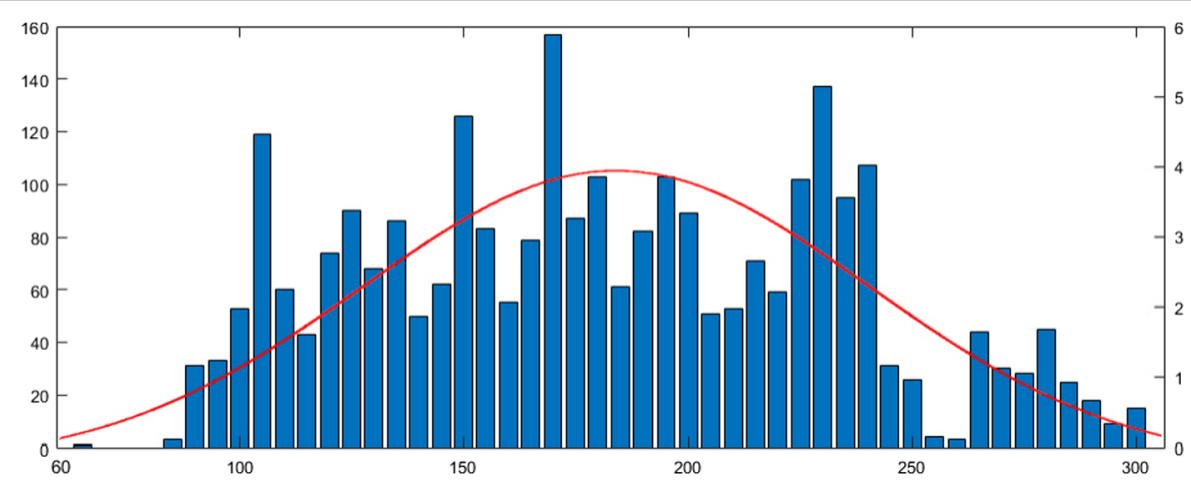

Figure 1 Flight connection time of passengers

\subsection{Model preparation}

\subsubsection{Partial data processing}

The flight transfer plan and transit passenger information includes the three days of January 19,20, and 21,2018. Only flights and passengers arriving on the 20th or departing on the 20th are analyzed here. Through MATLAB programming, the results show that there are 303 pairs of flights meeting the date requirements. There are 69 boarding gates in total. Thus, the results of boarding gates and flights are obtained through the above classification, as shown in Table 1 and Table 2 (X in the table refers to the mixed boarding gate, i.e. domestic flights and international flights can be docked).

The connection time of all flight tickets on 20th is counted, and the distribution of passenger flight connection time is shown in Fig. 1. For flights arriving on 20th or departing on 20th, if the arrival time and departure time are greater than or equal to 5 hours, stop the flight at the boarding gate after getting off the passengers, and stop the flight at the airport temporarily until the flight returns to the landing gate one hour before the flight (more than 45 minutes of free time between the two flights). Specific data of flights from boarding gate to temporary seat are shown in Table 3. The horizontal coordinate represents the flight connection time of passengers, and the left and right vertical coordinate respectively represents the corresponding number of passengers (people) and proportion $(\%)$.

\subsubsection{Entropy value method}

The weight is determined by the entropy value method based on personal preference [13]. The steps are as follows.

(1) non-negative data processing. 
Table 3 Partial data of flights from gate to temporary seat

\begin{tabular}{|c|c|c|c|c|c|c|c|c|c|}
\hline $\begin{array}{l}\text { Flight } \\
\text { pair }\end{array}$ & $\begin{array}{l}\text { Arrival } \\
\text { time }\end{array}$ & $\begin{array}{l}\text { Arrival } \\
\text { flight }\end{array}$ & $\begin{array}{l}\text { Departure } \\
\text { time }\end{array}$ & $\begin{array}{l}\text { Departure } \\
\text { flight }\end{array}$ & $\begin{array}{l}\text { Flight } \\
\text { pair }\end{array}$ & $\begin{array}{l}\text { Arrival } \\
\text { time }\end{array}$ & $\begin{array}{l}\text { Arrival } \\
\text { flight }\end{array}$ & $\begin{array}{l}\text { Departure } \\
\text { time }\end{array}$ & $\begin{array}{l}\text { Departure } \\
\text { flight }\end{array}$ \\
\hline PK062 & 19-14:55 & GN0523 & $20-16: 20$ & GN0256 & PK177 & 19-22:05 & NV6769 & $20-8: 35$ & NV612 \\
\hline PK072 & $19-15: 35$ & GN0497 & 20-17:05 & GN0644 & PK178 & $19-22: 10$ & GN945 & 20-9:40 & GN0476 \\
\hline PK089 & $19-16: 30$ & NV663 & 20-13:30 & NV692 & PK181 & $19-22: 25$ & GN0645 & 20-8:55 & GN0478 \\
\hline PK094 & 19-16:55 & NV693 & $20-13: 00$ & NV662 & PK183 & $19-22: 30$ & GN0639 & 20-9:10 & GN912 \\
\hline PK102 & $19-17: 35$ & GN0209 & 20-13:50 & GN0658 & PK184 & $19-22: 45$ & NV0747 & 20-9:05 & NV6446 \\
\hline PK112 & $19-18: 20$ & NV6253 & $20-10: 10$ & NV316 & PK185 & $19-22: 50$ & NV617 & $20-8: 55$ & NV6616 \\
\hline PK136 & 19-19:50 & NV6753 & 20-8:15 & NV6358 & PK186 & $19-22: 50$ & NV6379 & 20-9:50 & NV872 \\
\hline PK142 & $19-20: 10$ & GN0237 & $20-10: 40$ & GN920 & PK187 & $19-22: 55$ & NV6615 & $20-8: 25$ & NV6548 \\
\hline PK145 & $19-20: 10$ & NV6317 & 20-13:55 & NV6540 & PK189 & $19-23: 10$ & GN0285 & $20-9: 25$ & GN936 \\
\hline PK147 & $19-20: 15$ & NV6725 & $20-8: 20$ & NV6724 & PK190 & $19-23: 10$ & NV3464 & 20-10:15 & NV6160 \\
\hline
\end{tabular}

As the entropy value method based on personal preference [14] uses the ratio of a certain index of each scheme to the sum of the same index value, there is no dimensional influence and no need for standardized processing. If there are negative Numbers in the data, the data needs to be non-negative processing. In addition, in order to avoid the nonsense of logarithm when calculating entropy value, data translation is required.

For bigger is better and smaller is better, the indicators are

$$
r_{i j}=\frac{x_{i j}-x_{i}^{\min }}{x_{i}^{\max }-x_{i}^{\min }}+1 \quad \text { and } \quad r_{i j}=\frac{x_{i}^{\max }-x_{i j}}{x_{i}^{\max }-x_{i}^{\min }}+1 .
$$

Where, the nonnegative data is still $r_{i j}$.

(2) The proportion of scheme $j$ in index $i$ to this index $\varphi_{i j}$ is calculated by $R=\left(r_{i j}\right)_{m \times n}$.

$$
\varphi_{i j}=\frac{r_{i j}}{\sum_{i=1}^{m} r_{i j}}, \quad i=1,2, \ldots, m, j=1,2, \ldots, n .
$$

(3) The output entropy of the $i$ evaluation index $\varphi_{i j}$.

$$
\psi_{i}=-K \sum_{j=1}^{n} \varphi_{i j} \ln \varphi_{i j}, \quad j=1,2, \ldots, n .
$$

(4) Entropy weight coefficient of each target [15].

$$
w_{i}=\frac{1-H_{i}}{m-\sum_{i=1}^{m} H_{i}}, \quad i=1 .
$$

\subsection{Modeling}

Since the biggest impact of the newly added satellite hall is connecting flights of transfer passengers, the transfer intensity of transfer passengers is taken as the primary factor to establish the objective function. A multi-objective $0-1$ programming model is established, which is based on the following factors: minimum overall tension of passengers, minimum number of gates used, minimum number of flights not allocated to the gate and minimum number of passengers not successfully transferred. 


\section{Objective function.}

(1) The objective function to maximize the number of remaining boarding gates is

$$
\max F_{1}=\max X=\max \sum_{k=1}^{n} x_{k}
$$

Where, $x_{k}$ is a $0-1$ variable, indicating whether gate $k$ is used. The specific expression is as follows

$$
x_{k}= \begin{cases}1 & \text { gate } k \text { is not in use } \\ 0 & \text { gate } k \text { is used by flights. }\end{cases}
$$

(2) The minimum number of flights not allocated to the gate is

$$
\min F_{2}=\min \sum_{i=1}^{m} f_{i}
$$

Where, $f_{i}$ is a $0-1$ variable, indicating whether flight $i$ is allocated to the boarding gate. The specific expression is as follows

$$
f_{i}= \begin{cases}1 & \text { flight } i \text { is not assigned to the gate, } \\ 0 & \text { flight } i \text { has been assigned to the gate. }\end{cases}
$$

(3) Objective function with the objective of minimizing the overall tension of the transfer passengers.

Firstly, the formula of transfer tension is

$$
\text { Transfer tension }=\frac{\text { Passenger transfer time }}{\text { Flight connection time }},
$$

Passenger transfer time $=$ minimum process time

$$
+ \text { MRT time + walking time, }
$$

Connection time $=$ departure time of last flight

$$
\text { - arrival time of previous flight. }
$$

Secondly, transfer time and flight connection time can be expressed as follows.

Transfer time

$$
T_{1}=\sum_{i=1}^{m} \sum_{j=1}^{m} \sum_{k=1}^{n} \sum_{l=1}^{n}\left(c_{k l} y_{i k} y_{j l}+\mu_{k l} y_{i k} y_{j l}+\omega_{k l} y_{i k} y_{j l}\right) .
$$

Flight connection time

$$
T_{2}=D_{i k}-R_{j l}
$$


Finally, the objective function [16] established is as follows.

$$
\min \frac{T_{1}}{T_{2}}=\min \frac{\sum_{i=1}^{m} \sum_{j=1}^{m} \sum_{k=1}^{n} \sum_{l=1}^{n} p_{i j} \frac{\left(c_{k l} y_{i k} y_{j l}+\mu_{k l} l y_{i k} y_{j} l+\omega_{k l} y_{i k} y_{j l}\right)}{D_{i k}-R_{j l}}}{\sum_{i=1}^{m} \sum_{j=1}^{m} p_{i j}} .
$$

Where, $c_{k l}$ represents the overall minimum process time for tourists to enter from gate $k$ and exit from gate $l . c_{k l}$ is to match the elements in the matrix $C=\left(c_{k l}\right)_{69 \times 69}$ of the shortest process time between terminal $\mathrm{T}$ and satellite hall $\mathrm{S}$ with pairs of 69 boarding gates. $\mu_{k l}$ represents the MRT time when tourists enter from gate $k$ and leave from gate $l$, which is the element in MRT time matrix $\mu=\left(\mu_{k l}\right)_{69 \times 69} . \omega_{k l}$ represents the walking time of tourists entering from gate $k$ and exiting from gate $l$, which is the element in the walking time matrix $\omega=\left(\omega_{k l}\right)_{69 \times 69} \cdot y_{i k}$ is a $0-1$ variable, indicating whether flight $i$ is allocated to gate $k . D_{i k}$ represents the departure time of flight $i$ from gate $k . R_{j l}$ represents the arrival time of flight $j$ at gate $l$.

(4) The objective function [17] with the fewest number of unsuccessful passengers is

$$
\begin{aligned}
\min F_{4}= & \min \sum_{i=1}^{m} \sum_{j=1}^{m} \sum_{k=1}^{n} \sum_{l=1}^{n}\left[p_{i j}\left(1-y_{i k}\right)\left(1-y_{j l}\right)\right. \\
& \left.+p_{i j} y_{i k}\left(1-y_{j l}\right)+p_{i j}\left(1-y_{i k}\right) y_{j l}\right] .
\end{aligned}
$$

Where, $p_{i j}$ represents the number of passengers entering from flight $i$ and leaving from flight $j$.

\section{Constraint condition.}

(1) Applying the uniqueness of the gate used, the following constraints are established.

$$
\sum_{k=1}^{n+1} y_{i k}=1
$$

Where, the number of gates is $n+1$, and the extra 1 here represents the temporary slots set by the airport. $y_{i k}$ is a $0-1$ variable, indicating whether flight $i$ stays at gate $k$. The specific expression is

$$
y_{i k}= \begin{cases}1 & \text { flight } i \text { stops at gate } k \\ 0 & \text { flight } i \text { did not stop at gate } k .\end{cases}
$$

(2) Applying the flight model corresponding to the gate model (only domestic D and international I are considered here, regardless of the width/narrowness of the body), the constraints are

$$
H_{i} \in G_{j}
$$

Where, $H$ represents the set of flight models, with a total of 7 models, denoted as $H=\left\{H_{1}, H_{2}, \ldots, H_{t^{\prime}}\right\} . G$ represents the set of gate models, with a total of 10 models, denoted as $G=\left\{G_{1}, G_{2}, \ldots, G_{t^{\prime \prime}}\right\}$. Since there is a mixed gate situation, each element $G_{l}$ in set $G$ also represents a set, which refers to the set of flight models that gate $l$ allows to stop at. 
(3) In the statistical division of flight model and gate model, it is assumed that narrow flight model can be parked at the wide gate (wide flight model cannot be parked at the narrow gate) [18], which can greatly increase the use efficiency of the gate. The constraints are

$$
c_{a i}-c_{g k}+y_{i k} M \geq 0
$$

Where, the value of $c_{a i}$ can be 1 and 2, representing narrow and wide types of flights, respectively. Values of $c_{g k}$ can also be 1 and 2, indicating the narrow and wide gate models, respectively. $M$ is a big enough positive number.

(4) Applying the constraints of whether flight $i$ and $j$ can park at the same gate.

$$
\begin{aligned}
& \text { If }\left(D_{j k}-R_{i k}\right)\left(D_{i k}-R_{j k}\right)>0 \text {, we have } \\
& \qquad y_{i k}+y_{j k} \leq 1
\end{aligned}
$$

(5) Establish a constraint applying the safety interval time, that is, the interval between two adjacent flights parked at the same gate should be greater than or equal to the safety interval of 45 minutes, and the constraint is

$$
\left(1-z_{i j k}\right) M+R_{j k}-D_{i k} \geq 45
$$

Where, $R_{j k}$ represents the arrival time of the flight $j$ at gate $k . D_{i k}$ represents the departure time of flight $i$ from gate $k . z_{i j k}$ is a $0-1$ variable, indicating that when flights $i$ and $j$ are assigned to gate $k$ at the same time, whether $i$ is the previous flight that $j$ stops at the same gate $k$. The specific expression is

$$
z_{i j k}= \begin{cases}1 & i \text { is the previous flight that } j \text { stops at the same gate } k \\ 0 & \text { other situations. }\end{cases}
$$

Where, $z_{0 j k}=1$ represents stop at this gate on 19 th, leave on 20th and stop at gate $k$.

(6) Applying the constraints of the uniqueness of two consecutive flights at the same gate. That is, two consecutive flights at the same gate and one flight at the same gate.

$$
\sum_{k \in S} \sum_{i \in F} z_{i j k}=1, \quad y_{i k}+y_{j k}-2 z_{i j k} \geq 0 .
$$

The weight coefficient is determined as $w=(0.112,0.097,0.496,0.395)$ by entropy method [13] based on personal preference, and the final model [19] is obtained after weighted sum as follows

$$
\begin{aligned}
\max \left\{0.112 \sum_{k=1}^{n} x_{k}-0.097 \sum_{i=1}^{m} f_{i}\right. \\
-0.496 \frac{\sum_{i=1}^{m} \sum_{j=1}^{m} \sum_{k=1}^{n} \sum_{l=1}^{n} p_{i j} \frac{\left(c_{k l} y_{i k} y_{j l}+\mu_{k l} y_{i k} y_{j l}+\omega_{k l} y_{i k} y_{j l}\right)}{D_{i k}-R_{j l}}}{\sum_{i=1}^{m} \sum_{j=1}^{m} p_{i j}} \\
\left.-0.395 \sum_{i=1}^{m} \sum_{j=1}^{m} \sum_{k=1}^{n} \sum_{l=1}^{n}\left[p_{i j}\left(1-y_{i k}\right)\left(1-y_{j l}\right)+p_{i j} y_{i k}\left(1-y_{j l}\right)+p_{i j}\left(1-y_{i k}\right) y_{j l}\right]\right\},
\end{aligned}
$$


flight time conflict determination

flight assignment difficulty coefficient ranking

vertex assignmentcoloring

Figure 2 The flow chart of flight-gate assignment diagram coloring algorithm (a)

$$
\begin{cases}\text { s.t. } & \sum_{k=1}^{n+1} y_{i k}=1, \\ & H_{i} \in G_{j}, \\ & c_{a i}-c_{g k}+y_{i k} M \geq 0, \\ & y_{i k}+y_{j k} \leq 1, \\ & \left(1-z_{i j k}\right) M+R_{j k}-D_{i k} \geq 45, \\ & \sum_{k \in S} \sum_{i \in F} z_{i j k}=1, \\ & y_{i k}+y_{j k}-2 z_{i j k} \geq 0 .\end{cases}
$$

\section{Solution of model}

\subsection{Design of graph coloring algorithm based on parameter adjustment}

The flight-gate assignment graph coloring algorithm mainly includes three aspects [8]: flight time conflict determination, flight assignment difficulty coefficient ranking and vertex assignment coloring. The flow chart of flight-gate assignment diagram coloring algorithm is shown in Fig. 2.

Firstly, the concept of time slice is introduced into the determination of flight time conflict [9], and the time slice partition algorithm is adopted [4]. Secondly, "Difficulty coefficient" is adopted to measure the difficulty of seat allocation [8]. The vertex sequence number of each flight vertex can be obtained by sorting the difficulty coefficient of flight allocation. Flights are sorted in descending order applying vertex sequence number from largest to smallest, thus obtaining the assignment order of flights from difficult to easy. That is, the descending order of flight vertices corresponds to the assignment order of flights from difficult to easy. Where the difficulty coefficient is defined as $Q_{i}=\alpha \frac{H_{i}}{\bar{H}}+\beta \frac{\tau_{i}}{\bar{\tau}}+(1-\alpha-\beta) \frac{\kappa_{i}}{\bar{\kappa}}$. Finally, coloring algorithm [20] is used to color each flight vertex. In order to search for the flight allocation scheme with the least occupied gate, different $\alpha$ values are set repeatedly during the algorithm execution. By changing the value of $\alpha$, the weight of airframe types and conflicts in flight allocation can be adjusted, and the optimal scheme can be selected to reduce the number of gates occupied by flights.

Let the moment when flight $i$ stops using the gate be $F_{i}$, and all $F_{i}$ constitute the end time set $F=\left\{F_{i} \mid i=1,2, \ldots, N\right\}$. The specific algorithm steps are as follows.

Step 1. Set $\max F=0$ and initial values for $\alpha$ and $\beta(\alpha+\beta<1)$.

Step 2. Initialize the flight conflict table and calculate the flight assignment difficulty factor

$$
Q_{i}=\alpha \frac{H_{i}}{\bar{H}}+\beta \frac{\tau_{i}}{\bar{\tau}}+(1-\alpha-\beta) \frac{\kappa_{i}}{\bar{\kappa}}
$$




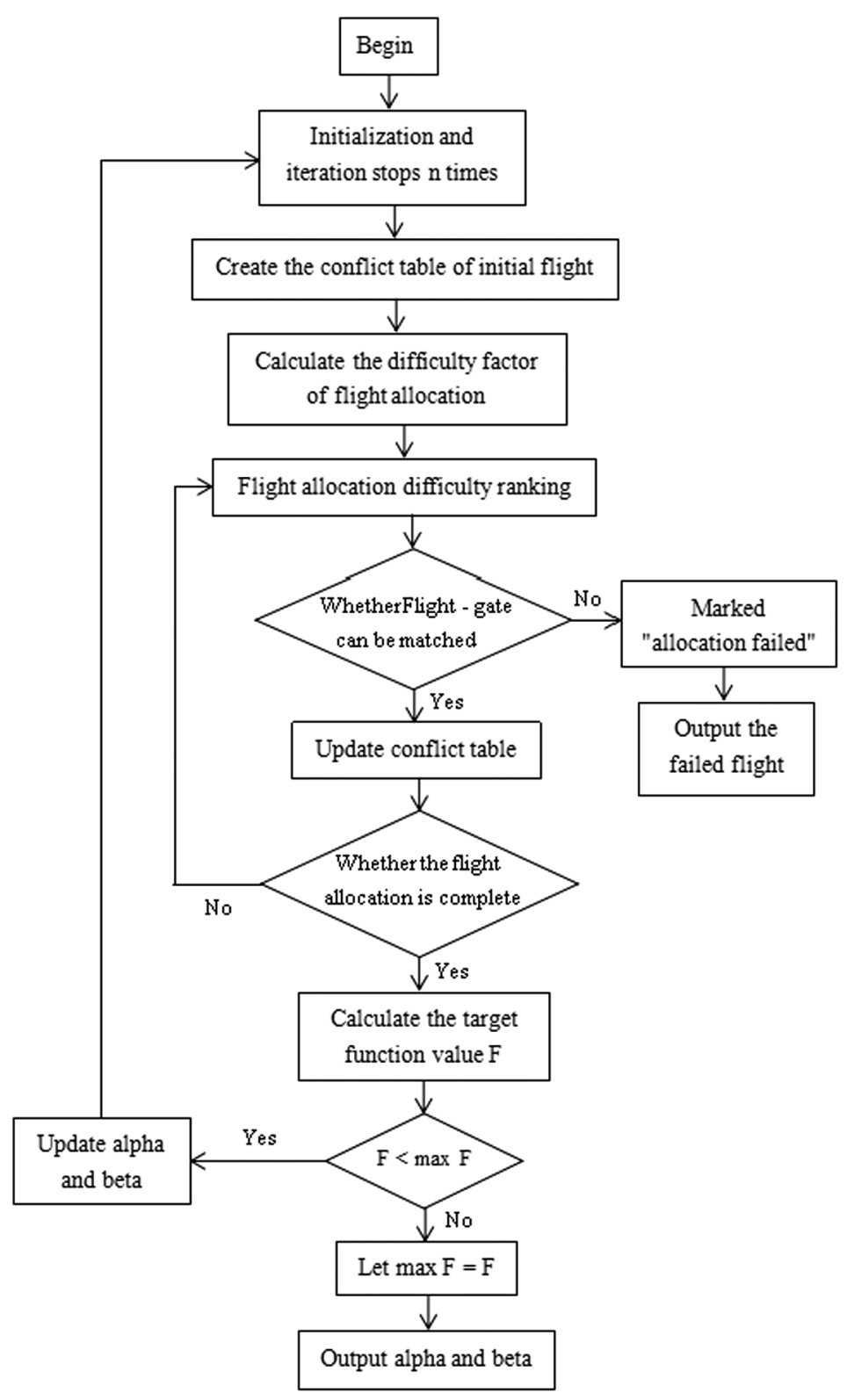

Figure 3 The flow chart of flight-gate assignment diagram coloring algorithm (b)

Step 3. Arrange the flight assignment difficulty factor and choose the largest $F_{i}$. If the largest is more than one, choose the first one by default.

Step 4. Go through all gate $G_{j}$ that can park flight $F_{i}$, and assign flights if no flights are scheduled in queue $G_{j}$. On the contrary, find the flight conflict table to determine whether there is conflict with existing flights. If there is no conflict, allocate it. Otherwise, it cannot be allocated, and mark it as "allocation failed".

Step 5. Update the flight conflict table, set the row and column of $F_{i}$ to 0 , and recalculate the flight assignment difficulty factor.

Step 6. If all flights have been allocated, turn to step 7. Otherwise, return to step 3. 


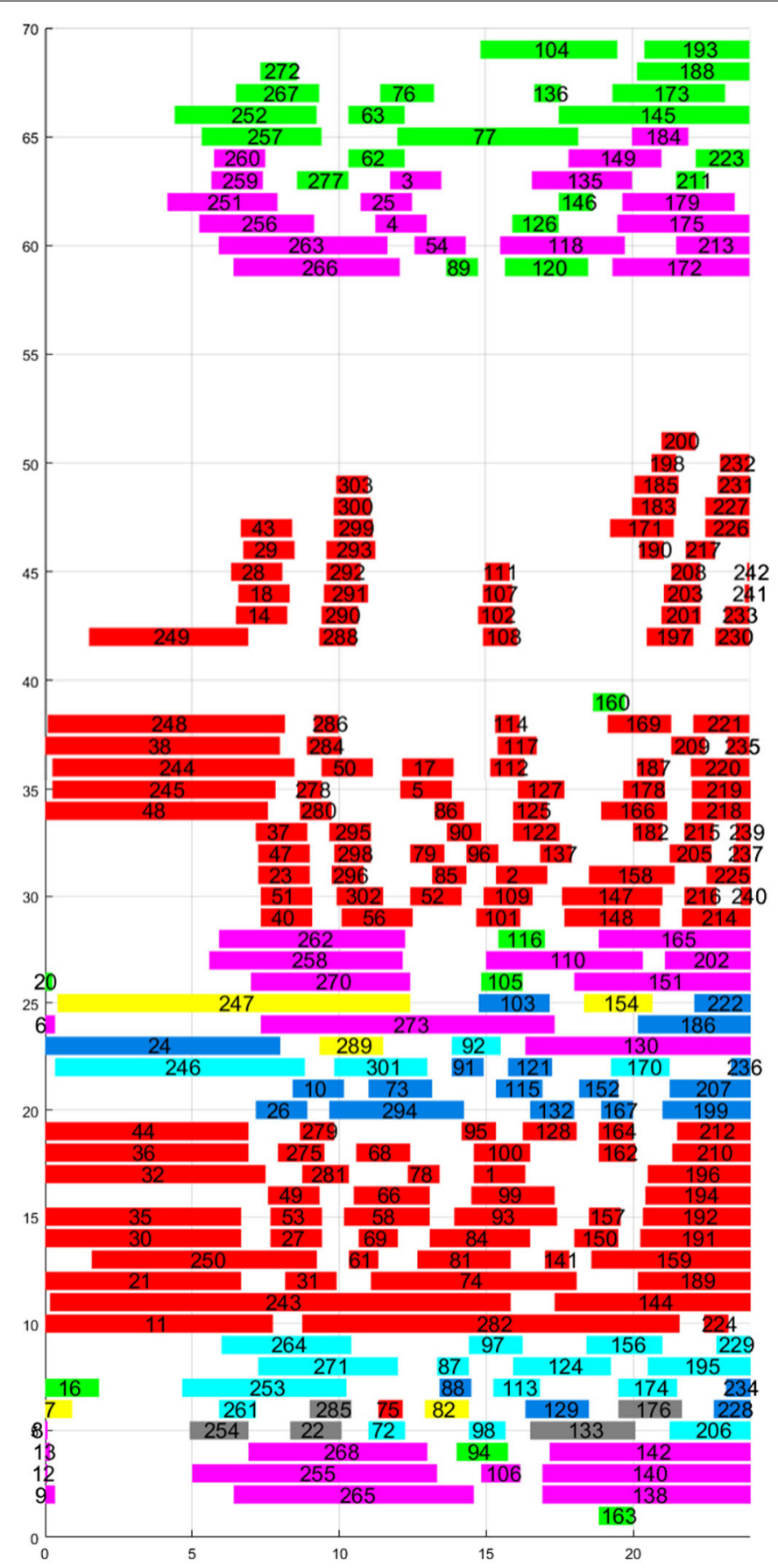

Figure 4 The flight assignment diagram

Step 7. Calculate the objective function value $F$, if $F<\max F$, turn to step 8. Otherwise, set $\max F=F$ and save the current values of alpha and beta.

Step 8. Stop after $n$ iterations, otherwise update the value of $\alpha, \beta$. Step size increases and $\beta$ remains the same, where $\alpha<1$ and $\beta<1-\alpha$. Return to step 2 .

The flow chart of the algorithm is shown in Fig. 3. 
Table 4 Partial data of the detailed schedule of the boarding gate

\begin{tabular}{llllllllll}
\hline $\begin{array}{l}\text { Boarding } \\
\text { gate }\end{array}$ & $\begin{array}{l}\text { Flight } \\
\text { pair }\end{array}$ & $\begin{array}{l}\text { Arrival } \\
\text { time }\end{array}$ & $\begin{array}{l}\text { Departure } \\
\text { time }\end{array}$ & $\begin{array}{l}\text { Flight } \\
\text { pair }\end{array}$ & $\begin{array}{l}\text { Arrival } \\
\text { time }\end{array}$ & $\begin{array}{l}\text { Departure } \\
\text { time }\end{array}$ & $\begin{array}{l}\text { Flight } \\
\text { pair }\end{array}$ & $\begin{array}{l}\text { Arrival } \\
\text { time }\end{array}$ & $\begin{array}{l}\text { Departure } \\
\text { time }\end{array}$ \\
\hline S31 & PK464 & $1 / 20-06: 25$ & $1 / 20-12: 05$ & PK370 & $1 / 20-19: 20$ & $1 / 21-00: 05$ & PK318 & $1 / 20-15: 40$ & $1 / 20-18: 30$ \\
S32 & PK461 & $1 / 20-05: 55$ & $1 / 20-11: 40$ & PK316 & $1 / 20-15: 30$ & $1 / 20-19: 45$ & PK411 & $1 / 20-21: 30$ & $1 / 21-09: 20$ \\
S33 & PK373 & $1 / 20-19: 30$ & $1 / 21-00: 10$ & PK454 & $1 / 20-05: 15$ & $1 / 20-09: 10$ & PK094 & $* * * * *$ & $1 / 20-13: 00$ \\
S34 & PK377 & $1 / 20-19: 40$ & $1 / 20-23: 30$ & PK159 & $* * * *$ & $1 / 20-12: 30$ & PK449 & $1 / 20-04: 10$ & $1 / 20-07: 55$ \\
S35 & PK333 & $1 / 20-16: 35$ & $1 / 20-20: 00$ & PK089 & $* * * *$ & $1 / 20-13: 30$ & PK457 & $1 / 20-05: 40$ & $* * * * *$ \\
S31 & PK287 & $1 / 20-13: 40$ & $1 / 20-14: 45$ & & & & & & \\
S32 & PK208 & $* * * * *$ & $1 / 20-14: 20$ & & & & & & \\
S33 & PK324 & $1 / 20-15: 55$ & $1 / 20-17: 30$ & & & & & & \\
S34 & PK344 & $1 / 20-17: 30$ & $1 / 20-18: 40$ & & & & & & \\
S35 & PK475 & $1 / 20-08: 35$ & $1 / 20-10: 20$ & PK409 & $1 / 20-21: 30$ & $1 / 20-22: 30$ & & & \\
\hline
\end{tabular}

\subsection{Solution of model}

The flight-gate allocation scheme and the use time of the gate are obtained by solving the flight-gate allocation graph coloring algorithm based on parameter adjustment. From the optimized flight-gate allocation diagram, it can be seen that the mean overall tension of the minimum transfer passengers is $35.179 \%$. The maximum number of flights successfully allocated to the gate is 262 pairs, and the minimum number of gates used is 60 . The corresponding flight-gate difficulty is assigned a weight $\alpha=0.32, \beta=0.40$, and the corresponding ratio of successful boarding gates is $86.469 \%$. The number of passengers who failed to transfer was 642 , with a failure rate of $23.337 \%$. The flight assignment diagram is shown in Fig. 4, in which different colors represent different flight models, the horizontal coordinate represents the flight waiting time length, and the vertical coordinate represents the gate number. Partial data of the detailed schedule of the boarding gate are shown in Table 4.

The number and proportion of flights successfully allocated to the gate are given by width and narrow-body aircraft respectively. It can be seen from the results in Fig. 5 that there are 49 and 213 flights with wide and narrow bodies respectively, and the probability of successful allocation of wide and narrow bodies is $100 \%$ and $83.858 \%$ respectively. The horizontal coordinate represents the body shape, and the left and right vertical coordinate respectively represents the successful allocation quantity and the successful allocation proportion.

After the optimization results are obtained, number of $\mathrm{T}$ and $\mathrm{S}$ gates used and average utilization rate of gates used (occupancy time ratio of gates) can be obtained by using MATLAB2018a software, as shown in Fig. 6. The horizontal coordinate represents the terminal hall, and the left and right vertical coordinate respectively represents the number of $\mathrm{T}$ and $\mathrm{S}$ gates used and the average utilization rate.

Figure 7 shows the time-occupancy ratio at 69 gates, with blue and red representing gates at $\mathrm{T}$ and $\mathrm{S}$ terminals, respectively. The horizontal coordinate represents the gate number, and the vertical coordinate represents the time-occupancy ratio.

The time distribution diagram of overall passenger transfer is drawn at the 5-minute transfer time interval, as shown in Fig. 8. The horizontal coordinate represents the transfer time, and the vertical coordinate represents the percentage.

The tension distribution diagram of overall passenger transfer is drawn at a tension interval of 0.1 , and the vertical coordinate represents the transfer passenger ratio, as shown in Fig. 9. The horizontal coordinate represents the tension, and the vertical coordinate represents the percentage. 

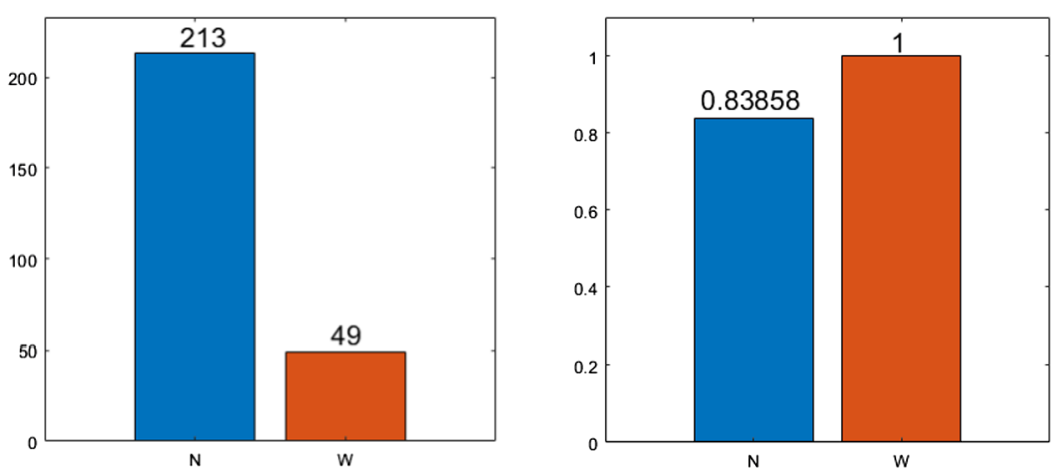

Figure 5 Number and probability of successful allocation of wide and narrow body machines
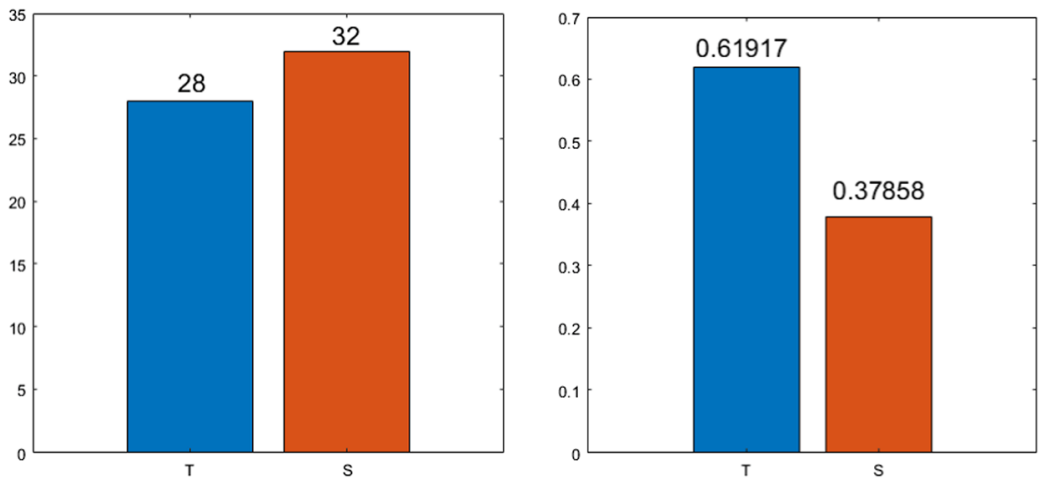

Figure 6 The number of T and S gates used and the average utilization rate

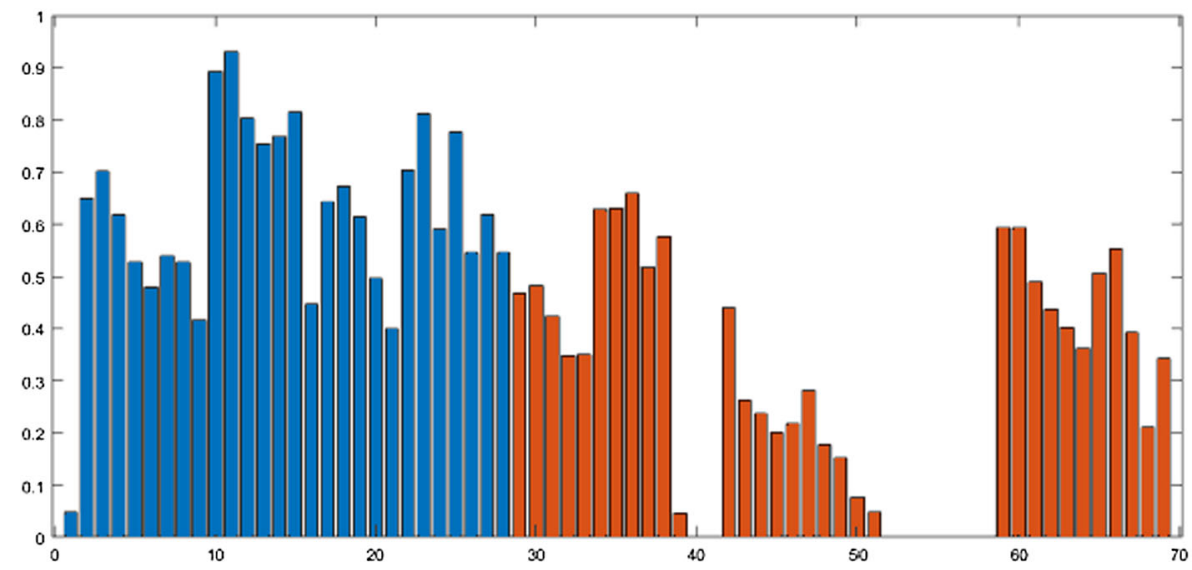

Figure 7 The time-occupancy ratio of each gate

\section{Conclusion}

In order to relieve the pressure of insufficient boarding gates in the original terminals, most airports have added satellite halls, which have a negative impact on connecting flights of transit passengers. The problem of flight-gate allocation for new satellite halls at airports mainly considers factors such as minimizing the number of gates used, minimizing the 


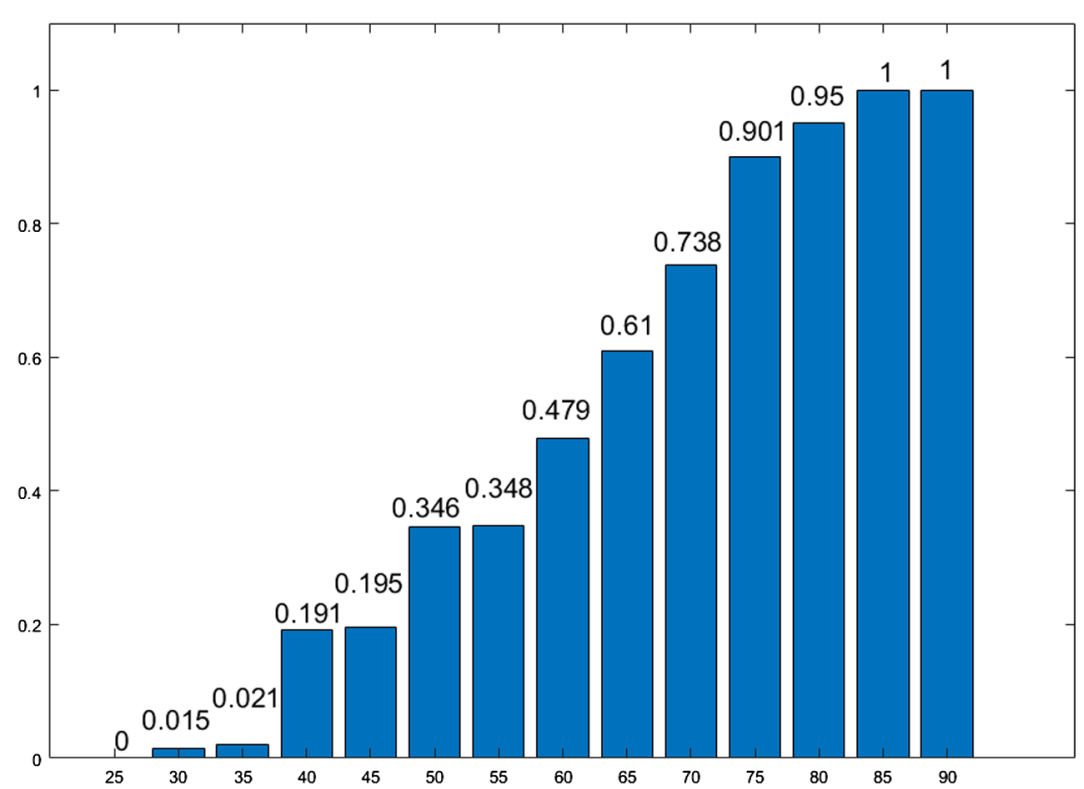

Figure 8 The time distribution diagram of transfer

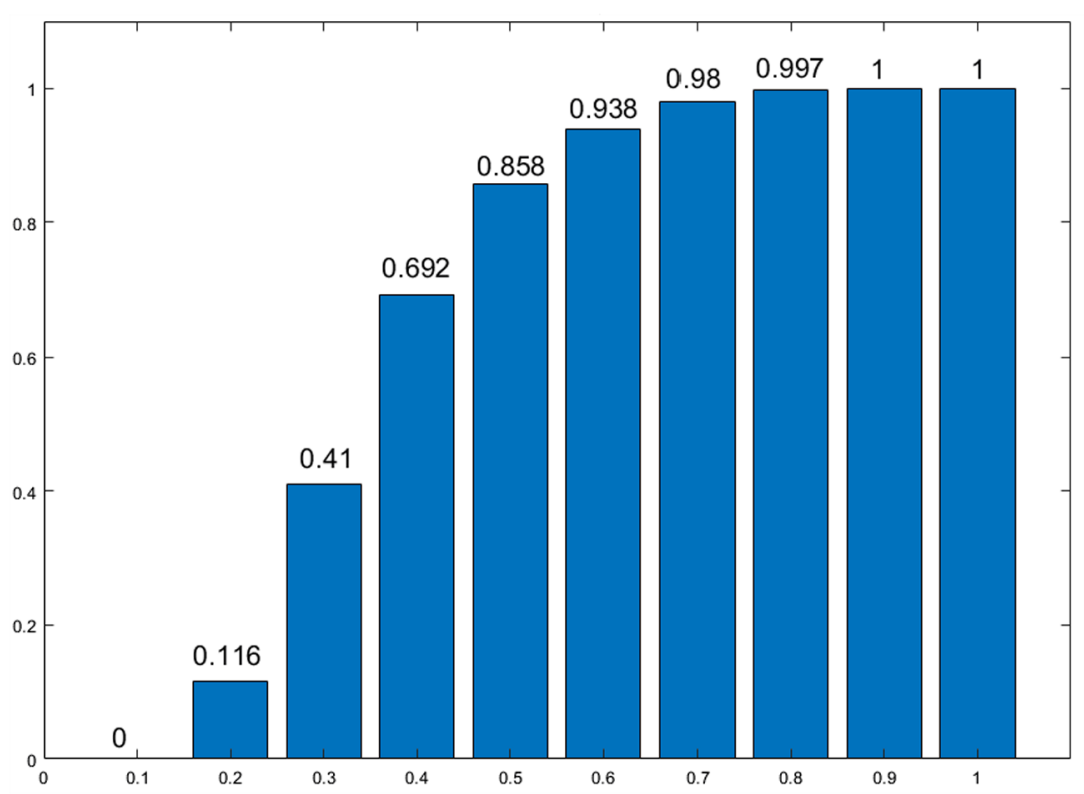

Figure 9 The tension distribution diagram

number of flights not allocated to the gate, minimizing the overall tension of passengers and minimizing the number of passengers not successfully transferred. A $0-1$ programming model based on entropy value method with personal preference was established. In the model solution, flight-gate assignment graph coloring algorithm based on parameter adjustment was used to obtain the flight assignment diagram, the detailed schedule of the boarding gate and the tension distribution diagram of overall passenger transfer. As can be seen from the obtained chart, the average overall tension degree of transfer passengers is 
$35.179 \%$, the number of flights successfully assigned to the boarding gate is 262 pairs, the number of gates used is 60, and the number of passengers who fail to transfer is 642, with a failure rate of $23.337 \%$. The results show that this model has great practical significance for solving the problem of flight-gate allocation.

\begin{abstract}
Acknowledgements
At the point of finishing this paper, l'd like to express my sincere thanks to all those who have lent me hands in the course of my writing this paper. First of all, l'd like to take this opportunity to show my sincere gratitude to my supervisor, $\mathrm{Mr}$ Xianfeng Ding, who has given me so much useful advices on my writing, and has tried his best to improve my paper. Secondly, I'd like to express my gratitude to my classmates who offered me references and information on time. Without their help, it would be much harder for me to finish this paper.
\end{abstract}

\title{
Funding
}

There is no funding for this research.

\section{Abbreviations}

MRT, Mass Rapid Transit; $Q_{i}$, The combined difficulty factor for flight $i ; \alpha, \beta(0 \leq \alpha \leq 1,0 \leq \beta \leq 1)$, Weight; $H_{i}$, Types of international/domestic flights; $\bar{H}$, Value of international and domestic flight crossover type; $\tau_{i}$, Wide/narrow body flight type; $\bar{\tau}$, The value of the wide-body flight type; $\kappa_{i}$, Number of conflicts between flight $i$ and other flights; $\bar{\kappa}$, Maximum number of time conflicts.

\section{Availability of data and materials}

The data used to support the findings of this study are available at the question $\mathrm{F}$ of the 2018 postgraduate mathematical contest in modeling website (https://cpipc.chinadegrees.cn/cw/hp/4). Please contact author for data requests.

\section{Competing interests}

The authors declare that they have no competing interests.

\section{Authors' contributions}

The main idea of this paper was proposed by LHY and DXF. LHY mainly carried out algorithm research and writing manuscripts. DXF is mainly responsible for supervising and revising papers. $L$ is mainly responsible for algorithm research and software. ZJY is mainly responsible for software programming. All authors read and approved the final manuscript.

\section{Author details}

${ }^{1}$ College of Science, Southwest Petroleum University, Chengdu, China. ${ }^{2}$ School of Computer Science, Southwest Petroleum University, Chengdu, China.

\section{Publisher's Note}

Springer Nature remains neutral with regard to jurisdictional claims in published maps and institutional affiliations.

Received: 30 June 2019 Accepted: 4 September 2019 Published online: 24 September 2019

\section{References}

1. Babic O, Teodorovic D, Tosic V. Air craft stand assignment to minimize walking. J Transp Eng. 1984;110(1):55-66

2. Mangoubi RS, Mathaisel DFX. Optimizing gate assignments at airport terminals. Transp Sci. 1985;19(2):173-88.

3. Yan S, Huo C. Optimization of multiple objective gate assignments. Transp Res, Part A, Gen. 2001;35(5):413-32.

4. Wen J, Li B, Wang Q, Wen D. Graph coloring model and algorithm for airport parking space allocation. Appl Syst Eng Theory Method. 2005;14(2):136-40.

5. Tian C, Xiong G. Airport gate position scheduling strategy based on genetic algorithm. Comput Eng. 2005;31(3):186-8.

6. Ju S, Xu L. Research on optimization problem of gate position assignment based on GSAA. J Transp Syst Eng Inf Technol. 2008;8(1):138-43.

7. Kong J. Research on modeling of airport gate position scheduling and genetic algorithm. 2008.

8. Jiang Y. Research on optimization of airport parking space allocation. Tianjin University. 2010.

9. Wen J. Genetic algorithm for airport gate position scheduling. Sci Technol Eng. 2010;10(1):135-9.

10. Li N. Simulation and optimization of airport gate allocation. Nanjing University of Aeronautics and Astronautics. 2013.

11. Zeng $L$, Jiang $Y$, Luo Y. Modeling of optimal assignment of gate position based on walking distance of passengers. J Wuhan Univ Technol. 2014;38(4):895-9.

12. Wang Y. Research on the mixed set planning method for airport gate position scheduling in busy airports. Nanjing University of Aeronautics and Astronautics. 2015.

13. Ruan $L$, Zheng X. Interval multi-objective decision making method based on entropy weight. Stat Decis Mak. 2013;12:82-4.

14. Zhang H, Zhao J, Luo H, Xie X. Objective weight calculation method for multi-objective optimization based on personal preference. Control Decis Mak. 2014;29(8):1471-6.

15. Zheng H, Li N, Yang X. Load balancing algorithm for adaptive load index weight. Comput Eng Des. 2019;40(03):623-43.

16. Liu S, Chen W, Liu J. Robust assignment of airport gates with operational safety constraints. Int J Autom Comput. 2016;13(01):31-41. 
17. Bouras A, Ghaleb MA, Suryahatmaja U.S., Salem A.M.. The airport gate assignment problem. Sci World J. 2014

18. Qin H, Liao Z. Multi-target athlete tracking method based on the characteristics of competition environment. Comput Eng Des. 2017;38(11):3173-8.

19. Li Q, Wang X. Multi-stage and multi-type equipment procurement decision-making model and solving algorithm. Comput Eng Des. 2016;37(11):3027-34.

20. Luo R, Xie R, Zhang D. Vertex shader model and algorithm for parking station allocation. Theory Pract Syst Eng. 2007;5(11):148-52.

Submit your manuscript to a SpringerOpen ${ }^{\circ}$ journal and benefit from:

- Convenient online submission

- Rigorous peer review

- Open access: articles freely available online

- High visibility within the field

- Retaining the copyright to your article

Submit your next manuscript at $\gg$ springeropen.com 